Revista de Psicología de la PUCP. Vol. XIV. No 1. 1996

\title{
DESARROLLO DEL JUICIO MORAL Y DE LA COMPLEJIDAD COGNITIVA A TRAVES DE UN DISEÑO INSTRUCCIONAL
}

Susana Frisancho'

El objetivo de esta investigación fué probar la eficiencia de un programa educativo (PECC) para elevar el nivel de razonamiento moral y de complejidad cogniriva. La muestra estuvo constituída por 18 estudiantes de 4to de secundaria, de ambos sexos, de 15 y 16 años de edad.

Los resultados indican que, aunque no se dieron avances estructurales en el nivel de razonamiento moral, hubo un incremento de la complejidad del razonamiento y en el número de elementos que los sujetos fueron capaces de identificar en los dilemas sociales.

Palabras claves: desarrollo moral, complejidad cogniriva, adolescentes, diseño instruccional, educación secundaria.

Moral judgement and cognitive complexity development through an instructional design The objective of this study was to test the efficiency of an educational program (PECC) in order to improve moral reasoning and cognitive complexity. The sample was 18 tenth-grade students of both genders, aged $15-16$ years.

Even though there were no structural advances in the moral reasoning level, there was an increase of reasoning complexity and in the number of elements that the subjects were able to identify in social dilemmas.

Keywords: Moral development, cognitive complexity, adolescents, instructional design, secondary education.

1. Magister en Psicología. Realizó sus estudios en la Pontificia Universidad Católica del Perú. Ha trabajado en el diseño de programas instrucionales. Se ha desempeñado como docente en la Sección de Psicología de la PUCP. Apartado 1761 Lima 100 - Perú. Correo electrónico: sfrisan@pucp.edu.pe. 



\section{Introducción}

Es ya un lugar común decir que el proceso educativo resulta fundamental en la formación moral de los niños y jóvenes, y que es la educación el espacio privilegiado para apoyar y fomentar el desarrollo de los valores y del pensamiento.

Internacionalmente la década del 90 ha sido considerada como la década de la calidad de la educación. Este énfasis en la calidad asume que la educación es un elemento fundamental a través del cual se puede igualar en términos de equidad y de justicia a las personas individuales $\mathrm{y}$ a las sociedades, y enfatiza el desarrollo de valores como el mejor camino para lograr una mejor convivencia entre las personas.

Una de las preguntas que con más insistencia se hace la educación se refiere al cómo dotar a los seres humanos de un sistema valorativo estable, sólido y flexible, que asegure la convivencia democrática así como el progreso de las sociedades. Sin embargo, a pesar de que el tema de la educación en valores es prioritario en la actualidad, aún no hay claridad ni coherencia en las diferentes propuestas que se dan en esta area. Los planteamientos generales y las declaraciones de principios (que crean el marco conceptual y las metas de la educación) no son suficientes para lograr el desarrollo efectivo de las personas, pues para cumplir con este propósito se necesita ir más allá de la simple declaración y llevar a cabo un trabajo específico en las escuelas, con los niños y jóvenes que van a conformar las instituciones sociales del futuro. En este sentido, la psicología, y específicamente la psicología del desarrollo moral, debe convertirse en una ciencia "de apoyo al desarrollo", que brinde modelos teóricos y propuestas educativas eficaces para entender y promover el desarrollo moral de las personas. 
Es lamentable reconocer que a pesar de la importancia que se le otorga a la educación moral y al desarrollo de valores como objetivos primordiales de la educación, en el Perú las escuelas todavía carecen de una estrategia de trabajo científica y efectiva para el cumplimiento de estos objetivos.

Los análisis y diagnósticos educativos realizados tanto en nuestro país como fuera de él concuerdan en indicar que existe una carencia a nivel moral y valorativo en la formación de los estudiantes. Las investigaciones muestran que los egresados de las escuelas secundarias tienen dificultades para razonar moralmente de manera compleja, y que presentan logros en el juicio y en el razonamiento inferiores a los que se esperarían para su edad.

Gran parte de los estudiantes de las escuelas secundarias e incluso de la universidad, en los que se esperaría encontrar niveles de autonomía moral, demuestran un desarrollo correspondiente a edades inferiores, y no muestran un uso de procesos cognitivos maduros. Si bien, es posible encontrar excepciones (vease por ejemplo el trabajo de Bello y Mejía, 1993), los diagnósticos educativos en nuestro país indican, en términos generales, que los niveles morales e intelectuales de nuestros jóvenes se encuentran por debajo de lo que podría esperarse en términos evolutivos.

La presente investigación intenta responder a este estado de cosas, planteando un programa educativo (PECC: Programa de Estimulación de la Complejidad Cognitiva), que puede ser insertado en el sistema educativo sin producir cambios demasiado bruscos que desorganicen su planificación, y que puede constituir un primer acercamiento a la educación moral en las escuelas. En esta investigación nos propusimos probar la eficacia del PECC, programa educativo que trabajando el razonamiento y là complejidad de pensamiento acerca de situaciones sociales problemáticas (dilemas morales) intenta incidir en el nivel de juicio moral de adolescentes de cuarto año de secundaria.

\section{Antecedentes}

Históricamente la educación moral ha mostrado dos grandes tendencias, las cuales han marcado la pauta en cuanto a programas de intervención y a la forma de entender el desarrollo moral y el aprendizaje de los valores. 
Tradicionalmente el sistema educativo concibió la enseñanza de la moral como la prédica de un conjunto de virtudes, reglas y costumbres basadas en la autoridad y la experiencia del maestro. En este modelo tradicional es el maestro el que posee las virtudes correctas, las cuales son inculcadas a los niños durante las clases normales o en reuniones especiales diseñadas para tal fin. Aunque esta forma de entender la educación moral se ha modificado de forma sustancial, aún subsiste la tendencia a educar a los niños con este método tradicional, el que ha tomado nueva forma en el enfoque llamado "educación del caracter", muy en voga en los Estados Unidos actualmente. Sin embargo, esta manera de asumir la educación en valores ha sido reconocida como ineficaz ya desde los años treinta (Lesser, 1967).

La segunda aproximación, usualmente asumida por escuelas de tendencia progresista, es el llamado método de clarificación de valores, que apunta a que cada persona reconozca en sí misma su propia escala y jerarquía de valores, y actue consecuentemente con ella. La versión inicial del método operaba bajo el supuesto del relativismo valorativo, aunque en versiones más recientes se asume que hay valores que son correctos.

Si bien esta forma de entender la enseñanza de la moral representa un avance con respecto a la anterior, resulta aún insuficiente, pues las investigaciones han demostrado que da resultados limitados dentro del contexto educativo (Lewis \& Miller, 1990).

La clarificación de valores intenta hacer a los alumnos conscientes de los suyos propios, pero deja de lado la toma de conciencia de los procesos de razonamiento moral que están a la base de dichos valores. Como afirma Reimer (1989), una persona puede identificar sus valores, pero continuar teniendo valores que no son (desde una perspectiva filosófica) racionalmente justificables. Sin embargo, y a pesar de las críticas recibidas, ambas metodologías, especialmente el método de inculcación, continuan siendo los métodos más utilizados como forma de educación moral en las escuelas.

En general, estas propuestas para el desarrollo de la moral dejan de lado el proceso evolutivo de cada individuo, pues se centran en inculcar o esclarecer un valor en sí sin tomar en cuenta el razonamiento que subyace a la elección de cualquier valor particular. Por lo tanto, se hacen pocas 
adaptaciones pedagógicas según la edad de los estudiantes, no se propicia la transferencia de lo aprendido a diferentes contextos y situaciones, ni se logran avances estructurales en el proceso de razonar.

$\mathrm{Al}$ analizar el currículo oficial, especialmente en educación secundaria, se encuentra un enorme vacío en el tema de la educación moral. De forma aislada aparecen en algunos cursos (principalmente religión y educación cívica) objetivos orientados a la formación de valores, aunque se trata solamente de objetivos de inculcación y no de desarrollo y razonamiento. Igualmente, los programas educativos de iniciativa particular apuntan principalmente a obtener logros específicos (es decir, intenta fomentar en los niños la adquisición de valores determinados, como el ser limpio, el ser honrado, etc.), desatendiendo las estructuras y los procesos de pensamiento que subyacen al desarrollo de la moral.

En esta investigación hemos asumido un modelo constructivista del desarrollo moral, entendiendo que la educación es un proceso que consiste en aportar las condiciones necesarias para permitir a las funciones psíquicas madurar a medida que se presentan, y pasar a funciones más elevadas de la forma más libre y completa. Por lo tanto, nos apartamos de los dos modelos tradicionales de enseñanza de la moral y proponemos una metodología de desarrollo basada en la teoría del desarrollo moral de Lawrence Kohlberg. Asumimos por lo tanto una orientación evolutiva, que desde hace varios años se ha planteado como alternativa tanto al modelo de inculcación como al de clarificación de valores.

Una de las formas metodológicas que esta orientación propone para la educación moral es la discusión de situaciones de conflicto sociocognitivo dentro del aula, de modo que se estimule el pensamiento de los estudiantes, el juego de roles, la toma de perspectiva y el análisis complejo de los dilemas morales. En esta investigación hemos adoptado esta metodología de trabajo.

\section{Marco conceptual}

Nuestro marco de referencia es la teoría del desarrollo cognitivo, específicamente el modelo kolhbergiano de desarrollo moral, al que se le ha agregado la variable diferencia individual, conceptualizada como el 
estilo cognitivo de complejidad/sencillez cognoscitiva. A continuación explicamos brevemente ambos conceptos.

\section{El modelo de desarrollo de Lawrence Kohlberg y sus aplicaciones educativas}

Lawrence Kohlberg es virtualmente el único psicólogo contemporáneo que reconoce que la filosofía resulta esencial para definir qué es la moral; este es el primer paso necesario para el estudio del desarrollo moral.

Kohlberg concluye que la estructura esencial de la moralidad es el principio de justicia; la esencia de este principio la encuentra en la distribución de deberes y derechos regulados por conceptos de igualdad y reciprocidad. Su interés fundamental es el estudio del razonamiento moral (y no los valores como entidades estáticas); son los factores de desarrollo que hacen que un individuo opte moralmente de una manera o de otra, que progrese desde un razonamiento heterónomo y poco elaborado, hasta otro de mayor grado de elaboración. En este proceso de desarrollo, se prioriza el componente cognitivo.

En sus investigaciones con chicos de entre 10 y 16 años, Kohlberg encontró que sus respuestas a dilemas morales hipotéticos podían ser clasificadas dentro de seis patrones de juicio moral distintos. Plantea entonces una secuencia de estadios de desarrollo en la cual cada estadio es prerequisito para la construcción del siguiente. Se identifican tres grandes niveles de desarrollo, dividos cada uno en dos estadios:

\section{Nivel preconvencional}

\section{Estadio 1:}

La acción moral se regula en función al castigo que se deriva de ella. Los deseos o mandatos de la autoridad se consideran órdenes sagradas. El regulador de la acción moral es fundamentalmente el castigo físico; no hay comprensión de que el castigo es una entre otras muchas consecuencias posibles de la conducta

\section{Estadio 2:}

Se inicia la coordinación de perspectivas y se dan pequeños avances en la toma de roles. Aparece el criterio de justicia que toma en cuenta 
las razones de la persona para ejecutar sus actos; esta concepción de justicia hace alusión a la igualdad entre las personas sin tomar en cuenta las diferencias específicas. Aparece la "Ley del Talión" (ojo por ojo y diente por diente), consecuencia del igualitarismo; el castigo debe responder exactamente al agravio recibido.

\section{Nivel convencional}

\section{Estadio 3:}

Se adquiere la habilidad de mirar la relación de dos personas desde la perspectiva de una tercera. En este período, el interés principal de las personas es vivir de acuerdo a las normas pre-establecidas, y satisfacer las expectativas de los otros individuos.

\section{Estadio 4:}

En este estadio el individuo pasa de compartir los puntos de vista de los otros individuos a tomar en cuenta el sentir del grupo social. Por primera vez se comprende a la sociedad como un sistema, apareciendo como prioritario el valor de la ley y el orden. El pensamiento moral se rige por los acuerdos sociales; cualquier acción que rompa estos acuerdos estaría rompiendo la estructura y la cohesión del grupo social.

\section{Nivel post convencional}

\section{Estadio 5:}

Este período hace referencia a un relativismo moral. Se piensa que los valores son relativos a determinado grupo social, pero la persona asume una posición para tratar de unificar las diferencias. Aparece así la noción de contrato social, entendido como un compromiso consciente por ambas partes a la que cada persona socialmente madura se obliga por inicitaiva propia.

\section{Estadio 6:}

Aparece la universalidad de los argumentos morales, priorizándose al ser humano como el valor fundamental al cual se somete todo lo demás.

Tardíamente, Kohlberg $(1980,1983)$ propone un estadio "suave" número 7 (en contraste con los seis estadios "duros" de su trabajo anterior), que delinea una orientación moral basada en un pensamiento ético y 
religioso, y envuelve una perspectiva y una vivencia cósmica y existencial de la vida y del mundo.

A nivel educativo, este modelo asume que la justicia no es un valor dado que pueda ser transmitido o impuesto a los niños, sino un principio moral, el proceso básico de valoración que subyace a la capacidad de cada persona para los juicios morales. Es este sentido de justicia el que, en cada estadio del desarrollo, da forma a los juicios que hacen los individuos sobre lo correcto y lo incorrecto. "Enseñar justicia", entonces, implica ayudar a los estudiantes a desarrollar e incrementar un más adecuado sentido de lo justo, ayudarlos a comprender mejor, y luego interiorizar, el principio de justicia en el cual se basa la filosofía moral de las normas y las leyes.

\section{El estilo cognitivo de complejidad/sencillez y su relación con el desarrollo moral}

La complejidad cognoscitiva es un estilo cognitivo que hace referencia a la capacidad que tienen las personas de representarse el mundo de manera pluridimensional, y se define en términos de dos procesos cognitivos variables: diferenciación e integración (Tetlock, Bernzweig \& Gallant, 1985).

La diferenciación se refiere a la variedad de aspectos o dimensiones del problema que el sujeto que toma la decisión reconoce, mientras que la integración alude al desarrollo de conexiones complejas entre estas características diferenciadas. La diferenciación es una condición necesaria para la integración, y constituye un proceso prerequisito para lograr el segundo.

Es posible establecer un continuo o gradiente de complejidad cognoscitiva, donde los extremos, marcadamente diferenciados, corresponderían a los puntos más altos de sencillez y de complejidad propiamente dicha. En este sentido, Mc Daniel \& Lawrence (1990) proponen un continuo teórico con los siguientes niveles de crecimiento de la complejidad cognitiva:

Nivel 1: Descripciones unilaterales

El estudiante simplifica la situación. Se centra en una sola idea o argumento, sin identificar alternativas. No trae nueva información, ni nuevos significados o perspectivas. Existe la tendencia a ver el mundo 
en categorías dicotómicas, lo que se traduce en aseveraciones del tipo "bueno/malo". Utiliza frases simples. Resta o repite información.

Nivel 2: Alternativas simples

Empieza a construirse el pensamiento causal, pero las explicaciones aún son simples. El estudiante toma una posición mediante no tomar en serio o ignorar las otras alternativas; esta posición es sustentada solamente mediante una idea o razón. Identifica conflictos simples y obvios, pero éstos no son analizados ni seguidos en el razonamiento. Defiende su posición con explicaciones simples más que con una evaluación profunda de la situación.

Nivel 3: Complejidad emergente

El estudiante identifica más de una posible explicación para los hechos; su nivel de explicación contiene al menos dos elementos o perspectivas. Establece y conserva esta complejidad. Introduce nuevos elementos, pero apoya su posición con comparaciones causales simples.

\section{Nivel 4: Interpretaciones amplias}

Hay mayor elaboración en el soporte de las ideas. Usa ideas extensas para definir e interpretar las situaciones. Se puede observar la introducción de una visión del mundo que no está contenida en la presentación del problema. De acuerdo a esta perspectiva establecida, el estudiante manipula sus ideas. Tiene un tema explicativo claramente reconocible. Puede integrar las ideas dentro de sub grupos, cada uno de ellos apoyando un componente de la explicación.

\section{Nivel 5: Análisis integrado}

Las respuestas exhiben una red causal que sintetiza la información y se apoya en evaluaciones globales e integradas. El estudiante construye una red de relaciones de causa efecto. Reestructura o reconceptualiza la situación, y se acerca al problema desde un nuevo punto de vista. Integra y extrapola ideas. Emplea metáforas, paralelos y analogías para aclarar la situación, llegando a través de ellas a nuevas interpretaciones. Aplica principios y hace generalizaciones del conocimiento del mundo. Elabora una estructura organizada, esboza conexiones y es capaz de predecir consecuencias. 


\section{¿Porqué vincular el desarrollo moral con la complejidad cognitiva?}

Al aceptar teoricamente que uno de los pilares fundamentales del desarrollo moral lo constituye la estructura cognitiva de los sujetos, asumimos también una directa relación entre el desarrollo moral y la complejidad de pensamiento.

Como afirma Kohlberg (1976), el razonamiento moral es claramente un razonamiento, y por lo tanto, un razonamiento moral avanzado depende de un razonamiento lógico avanzado, lo cual -entre otras cosas- implica manejar de manera ordenada y sistemática la mayor cantidad posible de información relevante con respecto a cada tema particular.

Desde nuestro punto de vista, la estructura cognitiva que subyace al razonamiento moral necesita de habilidades funcionales que le permitan desempeñarse optimamente. La complejidad cognitiva, al permitir un mayor éxito en la capacidad de identificar e integrar elementos de una situación social, tendría un rol de importancia en el proceso de razonar moralmente sobre dilemas sociales, ya que permitiría ubicar y organizar mejor la información necesaria para la elaboración de los juicios morales. Sugerimos que este factor de diferencia individual hará que los sujetos difieran en su estilo peculiar de resolver el conflicto interno que ocurre en una situacion moral (con mayor éxito para los individuos que logran razonar de forma compleja y menor éxito para los que razonan de forma simple).

En el desarrollo adolescente, los individuos van desde una forma de ver el mundo como una serie de categorías dicótomicas de correcto/ incorrecto (nivel de simplicidad cognoscitiva), hasta el entendimiento de que las decisiones y los compromisos deben ser hechos en un mundo marcado por falta de certeza y por imcompleta información (nivel de complejidad). Döbert \& Ninner-Winkler (1985) plantean que una de las metas del desarrollo moral es la habilidad de integrar aspectos del espacio social en argumentos morales "racionales". El sujeto que razona moralmente -afirman- eventualmente está inmerso en acciones concretas, contingencias y constreñimientos situacionales, roles, las consecuencias de la acción para uno mismo y para los demás o la sociedad, las intenciones que subyacen a las acciones y a las diferentes necesidades, tipos de motivos y 
motivaciones, el esquema del actor y las intuiciones sociales. Estos autores opinan que en algún momento el sujeto deberá identificar cada uno de estos factores, para finalmente ser capaces de integrar todos estos aspectos en una elaboración propia. Los autores afirman que incluso puede hablarse de diferentes estadios de desarrollo moral en función al avance en el desarrollo de esta complejidad cognitiva.

\section{Metodología}

\section{Características de la investigación}

Se trata de una investigación de nivel explicativo y de tipo cuasiexperimental, cuyo objetivo fue identificar los efectos del "Programa de Estimulación de la Complejidad Cognitiva" (PECC) sobre la complejidad cognitiva y sobre el razonamiento y el juicio moral. El diseño de la investigación es experimental, con un grupo de control equivalente sometido a pre-prueba y post-prueba.

\section{Objetivo y variables de investigación}

Nuestro objetivo fue identificar los efectos que tiene el "Programa de Estimulación de la Complejidad Cognitiva" (PECC) sobre la complejidad cognitiva y sobre el razonamiento y el juicio moral de un grupo de adolescentes de cuarto año de secundaria

\section{VI: Programa de Estimulación de la Complejidad Cognitiva (PECC)}

El "Programa de Estimulación de la Complejidad Cognitiva" (PECC) es un diseño instruccional elaborado para el trabajo con adolescentes, cuya meta es elevar el nivel de la complejidad cognitiva en relación a situaciones sociales.

El programa abarca 5 temáticas sociales: El aborto, el suicidio, la fidelidad, la eutanasia y la virginidad. Estos temas se identificaron a través de una encuesta diagnóstica de intereses y necesidades aplicada previamente con un grupo piloto de adolescentes. 


\section{VD: Juicio y razonamiento moral - Complejidad Cognitiva}

Razonamiento moral:

El razonamiento moral fue evaluado con el dilema de Heinz (traducido como dilema de Juan). Se usó la versión abierta de lápiz y papel, con calificación teórica.

Complejidad Cognitiva:

La complejidad cognitiva se refiere a la cantidad de factores identificados en una situación social, y a la integración de estos factores en el razonamiento y el juicio. Se evaluó mediante el análisis de contenido de las respuestas escritas en el dilema de Juan, calificadas según los criterios de Mc Daniel y Lawrence (1990).

\section{Muestra}

El tipo de muestreo fue no probabilístico, accidental. La muestra estuvo constituída por 18 estudiantes de cuarto de secundaria de un colegio particular alternativo de Lima Metropolitana, siendo 9 de ellos mujeres y 9 hombres. Las edades fluctuaron entre los 15 y los 16 años.

Los grupos experimental y de control fueron asignados aleatoriamente, quedando conformados de la siguiente manera:

Cuadro 1: Distribución de los sujetos en los grupos control y experimental

\begin{tabular}{|cccc|}
\hline & MASCULINO & FEMENINO & TOTAL \\
\hline GE & 5 & 4 & 9 \\
GC & 5 & 4 & 9 \\
\hline
\end{tabular}

\section{Instrumentos}

Los instrumentos utilizados fueron los siguientes:

1. Un diseño instruccional: "Programa de Estimulación de la Compleiidad Cognitiva" (PECC) 
2. Prueba de razonamiento y juicio moral y de complejidad cognitiva: Dilema de Juan, en versión abierta de lápiz y papel

3. Ficha de datos demográficos

4. Registro de eventos para la participación de los alumnos

5. Cuestionarios y situaciones sociales para la evaluación de logros en cada sesión y para la evaluación de la transferencia de habilidades

\section{PEEC: Descripción del programa}

El PECC es un diseño instruccional cuyo objetivo general es elevar el nivel de la complejidad cognoscitiva en relación a situaciones sociales. Se trabajan dos áreas específicas, a) la identificación de elementos de las situaciones sociales, y b) la integración de los elementos identificados. Estas areas se explican a continuación:

A. La identificación de los aspectos que conforman cada una de las situaciones sociales que se trabajan en el programa (aborto, fidelidad, eutanasia, suicidio y virginidad).

Esta área del programa apunta a brindar al estudiante contenidos diferenciados y distintas perspectivas para la observación de un mismo hecho social.

La tarea de identificación abarca 8 aspectos, que son los que propone el PECC en cada una de las situaciones sociales:

1. Naturaleza de la situación: tipo de transgresión o de conflicto social.

2. Rol propio: alude al papel del propio sujeto dentro del conflicto social.

3. Aspectos condicionantes: factores de la situación social relacionados a los hechos (edad del protagonista, situación socioeconómica real, etc.).

4. Consecuencias: efectos de la acción desarrollada. 
5. Alternativas: otros esquemas de acción frente a los desarrollados en la situación social.

6. Rol del vínculo: papel que juega el vínculo afectivo del propio participante y del protagonista de la situación (diferencias en el espacio afectivo).

7. Motivación del protagonista: razones psicológicas que pueden explicar el comportamiento del protagonista (no explícitas).

8. Intención del protagonista: razón explícita y objetiva por la cual el propio protagonista explica (o justifica) su acción.

B. Integración de los aspectos identificados en un razonamiento elaborado por el propio estudiante.

En esta área se retoma lo trabajado en la tarea anterior, y se trata de que el estudiante encuentre relaciones entre aquellos aspectos que ha identificado previamente. Para ello se utilizan diversas estrategias como el ejercicio de la toma de roles, el análisis de elementos y la realización de una síntesis conceptual de los elementos analizados.

\section{Procedimiento de aplicación del programa}

El PEEC se trabajó en un aula del colegio, con una frecuencia de una sesión semanal de dos horas de duración. Se tuvieron también tareas de trabajo para el resto de la semana, que los alumnos debieron hacer en sus casas (lecturas, etc.).

La aplicación del programa tomó 7 semanas, más dos semanas adicionales utilizadas para la evaluación inicial y final, lo que hace un total de 9 semanas de trabajo. El PECC se realizó bajo la conducción de una facilitadora, y con el apoyo de una persona que tomaba los registros de participación, apoyaba con los materiales y tomaba nota de información o sucesos relevantes.

Cada tema fue trabajado de forma independiente, y contó con su propio material para la evaluación de proceso. Al final de cada sesión se realizó una evaluación con situaciones de dilema moral vinculadas al tema trabajado. 
La transferencia de habilidades (de identificación de elementos y de integración) se evaluó con una situación social relativa a la pena de muerte, tema que no fué trabajado en el programa.

Antes de la aplicación del programa se realizó la evaluación inicial con el dilema de Juan, el cual volvió a utilizarse después de la última sesión. Esta evaluación se realizó con ambos grupos (control y experimental).

\section{Resultados y discusión}

Los resultados de las pruebas antes/después para los dos grupos (GE y GC) no indicaron variaciones en los puntajes obtenidos por los sujetos en complejidad cognitiva y en razonamiento moral.

Los niveles de complejidad cognitiva alcanzados por los sujetos variaron entre el nivel dos y el tres (alternativas simples y complejidad emergente respectivamente), sin que se diera un crecimiento en los niveles de complejidad cognitiva obtenidos en la primera y la segunda medición, para ninguno de los dos grupos.

Con respecto al nivel del juicio moral los estudiantes se ubicaron en los estadios 2 y 3 . Estos estadios corresponden al nivel Pre-Convencional (estadio 2) y al nivel Convencional (estadio 3). No hubieron cambios en el nivel del juicio moral, antes y después, en ninguno de los dos grupos.

Si bien de lo anterior podría deducirse que el programa no logró alcanzar su objetivo, el análisis cualitativo detallado de las respuestas de los sujetos nos indica que se dio un progreso en la cantidad de factores que los sujetos pudieron identificar en los problemas, así como en la complejidad de los argumentos utilizados en el razonamiento (aunque este progreso sea aún insuficiente para que los alumnos avancen de categoría o nivel). En base a los 8 aspectos que se trabajaron en el programa se encontró lo siguiente: 
Cuadro 2: Aspectos identificados en el dilema de Juan. Grupo experimental. (Cuadro resumen)

\begin{tabular}{|c|l|l|}
\hline $\begin{array}{c}\text { PARTI- } \\
\text { CIPANTE }\end{array}$ & \multicolumn{1}{|c|}{ PRE-TEST } & \multicolumn{1}{|c|}{ POST-TEST } \\
\hline 1 & $\begin{array}{l}\text { Naturaleza Vínculo Motivación } \\
\text { Condicionantes }\end{array}$ & $\begin{array}{l}\text { Vínculo Condicionantes } \\
\text { Consecuencias }\end{array}$ \\
\hline 2 & Consecuencias & $\begin{array}{l}\text { Consecuencias Motivación } \\
\text { Condicionantes }\end{array}$ \\
\hline 3 & Naturaleza Motivación & $\begin{array}{l}\text { Naturaleza Consecuencias } \\
\text { Motivación Alternativas }\end{array}$ \\
\hline 4 & $\begin{array}{l}\text { Alternativas Condicionantes } \\
\text { Naturaleza }\end{array}$ & $\begin{array}{l}\text { Consecuencias Condicionantes } \\
\text { Alternativas }\end{array}$ \\
\hline 5 & Alternativas Condicionantes & Consecuencias Condicionantes \\
\hline 7 & $\begin{array}{l}\text { Naturaleza Condicionantes } \\
\text { Vínculo Motivación }\end{array}$ & $\begin{array}{l}\text { Naturaleza Condicionantes } \\
\text { Motivación }\end{array}$ \\
\hline 8 & Nonsecuencias & $\begin{array}{l}\text { Vínculo Consecuencias } \\
\text { Condicionantes }\end{array}$ \\
\hline 9 & $\begin{array}{l}\text { Vínculo Consecuencias } \\
\text { Condicionantes }\end{array}$ & $\begin{array}{l}\text { Naturaleza Alternativas Vínculo } \\
\text { Condicionantes Motivación }\end{array}$ \\
\hline
\end{tabular}


Cuadro 3: Aspectos identificados en el dilema de Juan.

Grupo control. (Cuadro resumen)

\begin{tabular}{|c|c|c|}
\hline $\begin{array}{l}\text { PARTI- } \\
\text { CIPANTE }\end{array}$ & PRE-TEST & POST-TEST \\
\hline 1 & $\begin{array}{l}\text { Naturaleza Vínculo } \\
\text { Consecuencias }\end{array}$ & $\begin{array}{l}\text { Naturaleza Vínculo } \\
\text { Consecuencias }\end{array}$ \\
\hline 2 & Naturaleza Consecuencias & Motivación Consecuencias \\
\hline 3 & Alternativas Consecuencias & Alternativas Consecuencias \\
\hline 4 & Condicionantes Consecuencias & $\begin{array}{l}\text { Naturaleza Condicionantes } \\
\text { Consecuencias }\end{array}$ \\
\hline 5 & $\begin{array}{l}\text { Naturaleza Alternativas } \\
\text { Consecuencias }\end{array}$ & Alternativas Condicionantes \\
\hline 6 & Condicionantes Consecuencias & Condicionantes Consecuencias \\
\hline 7 & $\begin{array}{l}\text { Consecuencias Condicionantes } \\
\text { Motivación }\end{array}$ & Consecuencias \\
\hline 8 & Alternativas Consecuencias & Consecuencias Condicionantes \\
\hline 9 & $\begin{array}{l}\text { Vínculo Alternativas } \\
\text { Consecuencias Motivación }\end{array}$ & $\begin{array}{l}\text { Naturaleza Consecuencias } \\
\text { Condicionantes }\end{array}$ \\
\hline
\end{tabular}

El análisis cuidadoso de las respuestas de los sujetos nos revela diferencias en el número de aspectos identificados entre las respuestas del pre-test y las del post-test, principalmente en el grupo experimental. Por otro lado, observamos también un cambio cualitativo en los elementos identificados, pues aunque en algunos casos estos factores no aumentan cuantitativamente (es decir, no se identifican más factores), sí se cambian cualitativamente los aspectos observados en la situación, los puntos de vista o planos de observación. Esto quiere decir que aunque no se logró un avance en los niveles de complejidad cognitiva (por ejemplo, los adolescentes no progresaron del nivel 3 al 4), sí se dieron logros importantes en la habilidad para identificar elementos de una situación social, paso previo para el crecimiento de la complejidad de pensamiento. Esto nos lleva a pensar que con un mayor 
número de sesiones y mayor exposición al ejercicio de razonar, identificar elementos e integrarlos en una elaboración coherente, se hubieran logrado mayores avances en la complejidad del pensamiento.

Para explicar este hecho hipotetizamos que la memoria de trabajo juega un papel importante, pues existiría una capacidad limitada para asimilar diferentes puntos de vista en un determinado momento (tal como plantea por ejemplo Pacual-Leone cuando habla del "espacio mental M"); por lo tanto, los participantes-que reciben gran cantidad de información novedosa en el programa- reestructuran su pensamiento acerca de determinados tópicos, y al no poder manejar todos los nuevos planos de observación que se les ofrece, elaboran -y luego conservan- aquellos más novedosos o de más fácil asimilación (y por lo tanto cambian sus antiguos puntos de vista por otros nuevos, pero no aumentan la cantidad de planos de observación para una situación determinada).

Revisando el contenido de las respuestas encontramos que no todos los aspectos planificados en el PEEC para la identificación fueron alcanzados por los sujetos en sus razonamientos. De los ocho aspectos propuestos, solo se identifican seis, no llegándose a mencionar en las respuestas los factores "Rol Propio" e "Intención del Protagonista". La ausencia de estos elementos nos hace pensar en un escaso desarrollo de la habilidad de auto monitoreo y auto observación, ya que los participantes no han generado respuestas que indiquen una observación de sí mismos ocupando diferentes posiciones y roles. Así mismo, no se menciona la intención, elemento que tiene que ver la voluntad explícita de ejercer un efecto en el otro o en el medio en general, y de llevar a cabo, de forma explícita, una determinada acción o conducta.

Con respecto al juicio moral, los resultados nos han indicado que no hay diferencias significativas en el nivel del juicio moral de los sujetos de ambos grupos. Los estudiantes -tanto del grupo experimental como del grupo de control- se han ubicado en los estadios 2 y 3 de Kohlberg, que corresponden respectivamente al estadio de individualismo, propósito instrumental $\mathrm{e}$ intercambio, $\mathrm{y}$ al de expectativas interpersonales recíprocas, relaciones y conformidad interpersonal. El primero pertenece al nivel Pre Convencional (sub estadio B de ese nivel), mientras el segundo se ubica como parte del nivel Convencional (sub estadio A). Tales estadios resultan esperables para el grupo de edad con el que se trabajó. 
Cuadro 4: Argumentos sobre la vida humana en relación a la esposa. Grupo experimental

\begin{tabular}{|c|c|c|c|}
\hline $\begin{array}{l}\text { PARTICI- } \\
\text { PANTE }\end{array}$ & $\begin{array}{l}\text { ¿QUE DEBERIA } \\
\text { HACER JUAN? }\end{array}$ & $\begin{array}{l}\text { RAZONES QUE } \\
\text { ARGUMENTAN } \\
\text { LA RESPUESTA* }\end{array}$ & $\begin{array}{c}\text { ¿QUE OCURRE } \\
\text { SI JUAN NO } \\
\text { AMA A SU ESPOSA?* }\end{array}$ \\
\hline 1 & Robar & $\begin{array}{l}\text { Tiene que hacer todo lo po- } \\
\text { sible por salvar a su esposa, } \\
\text { por buen esposo que es / Lo } \\
\text { anterior más: lo que el farma- } \\
\text { céutico está haciendo es in- } \\
\text { justo. }\end{array}$ & $\begin{array}{l}\text { Si Juan no la ama no tiene } \\
\text { que robar. }\end{array}$ \\
\hline 2 & No robar & $\begin{array}{l}\text { Si lo atrapan lo matan / el } \\
\text { esposo se quedaría solo. }\end{array}$ & $\begin{array}{l}\text { Si no la quiere no tiene } \\
\text { obligación de ayudarla. }\end{array}$ \\
\hline 3 & Robar & $\begin{array}{l}\text { Está en juego una vida hu- } \\
\text { mana / Lo anterior más: In- } \\
\text { tentó juntar la plata y no } \\
\text { pudo. }\end{array}$ & $\begin{array}{l}\text { Es un ser humano, ya sea } \\
\text { su esposa, el vecino, su hijo } \\
\text { etc. }\end{array}$ \\
\hline 4 & No Robar & $\begin{array}{l}\text { Está violando la ley, es un } \\
\text { pecado / estaría metiéndose } \\
\text { en otro problema, podrían } \\
\text { descubrirlo; la medicina es de } \\
\text { la otra persona. }\end{array}$ & Es lo mismo. \\
\hline 5 & Robar & $\begin{array}{l}\text { No tiene otra salida para sal- } \\
\text { var a su esposa. }\end{array}$ & $\begin{array}{l}\text { Así le demostraría que } \\
\text { aunque no la ame es im- } \\
\text { portante en su vida. }\end{array}$ \\
\hline 6 & Robar & $\begin{array}{l}\text { Para salvar la vida de su es- } \\
\text { posa; además el farmaceútico } \\
\text { está especulando. }\end{array}$ & $\begin{array}{l}\text { La vida de cualquiera vale } \\
\text { mucho. }\end{array}$ \\
\hline 7 & Robar & $\begin{array}{l}\text { Ama a su esposa y no tiene } \\
\text { suficiente dinero. }\end{array}$ & $\begin{array}{l}\text { Que la ayude porque él se } \\
\text { senti-ría bien después de } \\
\text { salvarla sin amarla. }\end{array}$ \\
\hline 8 & Robar & $\begin{array}{l}\text { Lo más importante es salvar } \\
\text { una vida. }\end{array}$ & $\begin{array}{l}\text { Aunque no la ame ella } \\
\text { también tiene sentimien- } \\
\text { tos y quiere vivir. }\end{array}$ \\
\hline 9 & $\begin{array}{l}\text { Robar / No } \\
\text { puede decidir }\end{array}$ & $\begin{array}{l}\text { La vida humana es lo más im- } \\
\text { portante y el farmaceútico es } \\
\text { egoísta / Hay demasiados as- } \\
\text { pectos importantes que pue- } \\
\text { den suceder y que hay que } \\
\text { tomar en cuenta. }\end{array}$ & $\begin{array}{l}\text { Es importante salvar a un } \\
\text { ser humano aunque en } \\
\text { este caso, ya que no la ama, } \\
\text { no debe llegar a robar. }\end{array}$ \\
\hline
\end{tabular}

* Salvo cuando se indique lo contrario, las respuestas son las mismas en el Pre y el Post-Test. Cuando existen diferencias, las respuestas del Post-Test aparecen después del signo \% 
A continuación ofrecemos, a manera de ejemplo, algunas de las respuestas dadas por los estudiantes al dilema de Juan:

Cuadro 5: Argumentos acerca de la vida humana en caso de un amigo o un extraño. Grupo experimental

\begin{tabular}{|c|c|c|}
\hline $\begin{array}{l}\text { PARTICI- } \\
\text { PANTE }\end{array}$ & $\begin{array}{l}\text { ¿QUE OCURRE SI SE TRATA } \\
\text { DE VIDA DE UN AMIGO? }\end{array}$ & $\begin{array}{l}\text { ¿QUE DEBERIA HACER JUAN } \\
\text { EN EL CASO DE UN EXTRAÑO? }\end{array}$ \\
\hline 1 & $\begin{array}{l}\text { Hay que robar porque la amistad } \\
\text { es lo mejor que puede suceder en } \\
\text { la vida de una persona. }\end{array}$ & $\begin{array}{l}\text { No hay que ayudarlo porque } \\
\text { puede ser un ladrón o un vulgar. }\end{array}$ \\
\hline 2 & No responde. & $\begin{array}{l}\text { No robar, puede ser una broma. } \\
\text { Hoy en día no se puede confiar } \\
\text { en nadie y menos en un extraño. }\end{array}$ \\
\hline 3 & $\begin{array}{l}\text { Una vida es muy valiosa para to- } \\
\text { dos y no podemos dejar que una } \\
\text { persona muera así porque sí. }\end{array}$ & $\begin{array}{l}\text { Igual habría que salvarlo, no po- } \\
\text { demos dejar tan friamente que } \\
\text { muera una persona. }\end{array}$ \\
\hline 4 & $\begin{array}{l}\text { Es una persona igual que todos y } \\
\text { aún vale más si es un amigo. }\end{array}$ & Hay que ayudarlo pero sin robar. \\
\hline 5 & $\begin{array}{l}\text { Hay que ayudarlo porque si es un } \\
\text { verdadero amigo también haría eso } \\
\text { por Juan. }\end{array}$ & $\begin{array}{l}\text { Depende de los recursos del ex- } \\
\text { traño (si tiene dinero, etc.). }\end{array}$ \\
\hline 6 & $\begin{array}{l}\text { Si el amigo recurre a Juan es por- } \\
\text { que no tiene a nadie más. Hay } \\
\text { que ayudarlo porque no es una } \\
\text { enfermedad sencilla, es algo grave. }\end{array}$ & $\begin{array}{l}\text { Igual lo ayudaría para sentirme } \\
\text { bien conmigo. ¿Que pasaría si yo } \\
\text { estuviese en su lugar?. }\end{array}$ \\
\hline 7 & $\begin{array}{l}\text { Hay que hacer lo posible por ayu- } \\
\text { dar a nuestros amigos. }\end{array}$ & $\begin{array}{l}\text { La persona necesita la medicina, } \\
\text { hay que robar para salvarlo. }\end{array}$ \\
\hline 8 & $\begin{array}{l}\text { Los amigos deben ayudarse en las } \\
\text { buenas y en las malas, es una ayu- } \\
\text { da mutua. }\end{array}$ & $\begin{array}{l}\text { Hay que salvarlo ya que más ade- } \\
\text { lante él también te ayudará. }\end{array}$ \\
\hline 9 & $\begin{array}{l}\text { No sé, pero pienso que no se puede } \\
\text { hacer morir a una persona cerca- } \\
\text { na. }\end{array}$ & $\begin{array}{l}\text { No hay que ayudarlo, no se sabe } \\
\text { quién es ni de qué sufre / no puedo } \\
\text { decidir, pero creo que no es im- } \\
\text { portante salvarlo. }\end{array}$ \\
\hline
\end{tabular}


Analizando las respuestas vemos claramente que el valor de la vida presenta características conceptualizadas por Kohlberg como típicas de los estadios 2 y 3 . El valor de la vida para el estadio 2 es un valor inmediato para el esposo y aún para la propia esposa; se asume que la vida de la esposa es suficientemente importante para que Juan robe por ella, pero no está obligado a hacerlo para salvar a un amigo, y mucho menos aún por un extraño. El valor de la vida depende entonces de la relación personal establecida. Estas respuestas se hacen evidentes en aquellos participantes que seńalan que no se debe ayudar a un desconocido, supeditando esta ayuda a la "calidad" de la persona (si el extraño tiene recursos, si no es un vulgar, si es alguien importante, etc.). Vemos pues que no hay una noción trascendente de vida humana, sino representaciones concretas, ligadas a elementos particulares que supeditan este valor a características de la persona o de la relación (vínculo) que con ella se establece.

En el estadio 3, la orientación básica "buena niña/buen niño" hace que el valor de la vida se conceptualice en estos mismos términos; la vida es un valor de toda persona buena, como lo es Juan. Aquí el esposo quiere y cuida a su esposa y la quiere lo suficiente para robar por ella. De igual forma, un ser humano bueno se preocupa por salvar a su amigo y a cualquier otra persona. En las respuestas de los estudiantes vemos esta posición, por ejemplo, cuando se dice que Juan debe salvar a su esposa "por buen esposo que es". Esto también puede verse cuando se afirma que se debe ayudar al otro para sentirse bien con uno mismo, apelando otra vez al estándar de "buena persona", cuyo deber es ayudar a los demás (el cumplir con una obligación propia del estándar hace que la persona se evalue positivamente, y como consecuencia, que se sienta bien con ella misma).

En las respuestas de los sujetos hemos encontramos también elementos de reciprocidad, pues en muchas de ellas se señala explicitamente la importancia de ayudar para poder después ser ayudado. Este elemento de reciprocidad es también característica de los estadios de desarrollo más elementales.

$\mathrm{Si}$ bien en algunas respuestas aparecen elementos que podríamos considerar propios de un estadio 4 (por ejemplo, el valor de la vida como algo general, un "carácter sagrado" de la vida humana) consideramos que 102 
el nivel mayoritario en el que se ubica el sujeto continua siendo el 3, debido a las razones o argumentaciones dadas en función de esta concepción de vida humana. Así, vemos que aún cuando se señala un valor general de la vida, ésta continúa concibiéndose como una función individual, producto de los sentimientos o las responsabilidades humanas, y no como producto de un consenso social o de una jerarquía de derechos. En todo caso, planteamos que estas mixturas en los razonamientos pueden ser indicadores de una estadio de transición.

Una vez que hemos descrito los resultados que se encontraron en la evaluación inicial, final y de proceso del PECC, es importante intentar dar una explicación que permita entender las posibles razones de estos resultados, a la vez que plantear sugerencias para futuros trabajos.

Dado que los resultados indican que sí hubieron logros a nivel de pensamiento, a pesar del reducido número de sesiones (y que por lo tanto programas educativos similares al PECC podrían dar buenos resultados en los colegios), se hace necesario reelaborar el programa para darle mayor duración e intensidad (más sesiones por semana y más semanas de trabajo en el año escolar). Esto redundará en un mayor y mejor desarrollo tanto de la complejidad cognitiva como del nivel de razonamiento y juicio moral. Es importante recordar que el avance estructural y el desarrollo es un proceso contructivo, que requiere de duración en el riempo y de constancia $\mathrm{e}$ intensidad de la estimulación. Igualmente, se sugiere ampliar los temas trabajados a otros que sean de interés de cada grupo de estudiantes en particular.

Es también importante tener en cuenta variables de modalidad sensorial (utilizar materiales de varias modalidades y no priorizar una sola), para favorecer a todos los estudiantes en sus estilos de aprendizaje. Al ser el programa netamente verbal (pues se incorporaron materiales escritos y se razonó y discutió a través del lenguaje), el lenguaje y la inteligencia verbal son factores importantes. Sabemos que la experiencia humano-social $-\mathrm{y}$ por consiguiente la experiencia moral-no podría asimilarse si el hombre careciera de lenguaje; a través del lenguaje el niño se adueña de un nuevo factor de desarrollo -la adquisición de la experiencia humano-social- que se convierte rapidamente en el factor fundamental de su formación mental. 
Pensamos por lo tanto que un buen nivel de desarrollo lingüístico podría estar asociado a un mejor desempeño en programas como el PECC.

Una de las variables que consideramos más relevantes para la comprensión de los resultados es el nivel operatorio de los sujetos. Al ser el juicio moral una macro estructura, que abarca a operaciones más fundamentales (clasificación, seriación, e incluso conservación y causalidad), el nivel que el sujeto haya alcanzado en estas operaciones sería un buen predictor del desarrollo que podría alcanzar en el juicio moral. Recordemos que Kohlberg plantea que no es posible alcanzar un desarrollo moral superior al desarrollo lógico; si las operaciones lógicas no están desarrolladas, tampoco podrá estarlo el juicio moral, y se produciría un desajuste entre las posibilidades de asimilación de la información de que disponen los sujetos y los niveles de razonamiento que requiere el tipo de tareas que deben realizar.

Para finalizar, podemos decir los temas tratados en las sesiones y el tipo de tareas que el programa demandó a los sujetos no son cuestiones sencillas. Es importante considerar el papel del objeto cuando se quiere hacer estimulación operatoria; en una macro operación como es el juicio moral, no se trata ya de objetos estáticos sobre los cuales el sujeto opera de manera individual, sino de una interacción compleja con un/unos objetos que dejan de ser pasivos y se convierten también en sujetos de acción. No es una interacción con el mundo físico, sino con el complejo mundo social.

\section{Referencias}

Asendorpf, Jens B. y Nunner-Winkler, Gertrud (1992). Children's Moral Motive Strength and Temperamental Inhibition Reduce their Immoral Behavior in Real Moral Conflicts. Child Development, 63 (5), 12231235.

Bello, M. y Mejía, E. (1993). Relaciones entre el nivel de desarrollo moral $e$ intelectual, edad y género en alumnos de secundaria de un colegio experimental/alternativo de Lima. Revista de Psicologia, Lima: PUC, 11 (1), 13-30.

Blasi, A. (1980). Bridging Moral Cognition and Moral Action: A Critical Review of the Literature. Psychological Bulletin, 88, 1-45. 
Bruner, J. (1988). Desarrollo cognitivo y educación. Madrid: Ediciones Morata.

Cueto, S. y Mueller, D. (1990). Razonamiento Moral en Cinco Niveles Educativos. Revista de Psicología, Lima: PUC, 8 (1), 11-20.

Darley, John M. y Shultz, Thomas R. (1990). Moral Rules: Their Content and Acquisition. Annual Review of Psychology, 41.

Döbert, R. y Nunner-Winkler, G. (1985). Ińterplay of Formal and Material Role Taking in the Understanding of Suicide Among Adolescents and Young Adults. I: Formal and Material Role Taking. Human Development, 28 (5).

Döbert, R. y Nunner-Winkler, G. (1985). Interplay of Formal and Material Role Taking in the Understanding of Suicide Among Adolescents and Young Adults. II: Naive Suicide Theories and Structural Approach. Human Development, 28 (6).

Hageseth, Jon A. (1983). Relationships Among Cognitive Complexity Variables. Psychological Reports, 52 (2).

Hersh, R.; Paolitto, D. y Reimer, J. (1984). El Crecimiento Moral. De Piaget a Koblberg. Madrid: Narcea Ediciones.

Kohlberg, Lawrence (1976). Moral Stages and Moralization. The Cognitive Developmental Approach En: Lickona, Thomas. Moral Development and Behavior. Theory, research and social issues. Nueva York: Holt, Rinehart y Winston Editores.

Kurtines, William (1986). Moral Behavior as a Rule Governed Behavior: Person and Situation Effects on Moral Decision Making. Journal of Personality and Social Psychology, 50 (4), 784-791.

Kurtines, William M. \& Gewirtz, Jacob L. (1984). Morality, Moral Behavior and Moral Development. A Wiley-Interscience Publication. Nueva York: John Wiley \& Sons.

Lesser, Gerald (1981). La Psicología en la Práctica Educativa. México: Trillas.

Lewis, Michael y Miller, Suzanne M. (1990). Handbook of Developmental Psychopatology. Nueva York: Plenum Press.

Luria, A.R.; Leontiev, A.N. y Vigotsky, L.S. (1986). Psicologia y Pedagogia. Madrid: Ediciones Akal.

Mc Daniel, E. y Lawrence, C. (1990). Levels of Cognitive Complexity. An Approach to the Measurement of Thinking. Nueva York: SpringerVerlag. 
Reátegui, N.; Frisancho, S. y Del Valle, R. (1993). Estudio diagnóstico del funcionamiento académico de los estudios generales letras y del perfil psicológico de los estudiantes. Documento de trabajo. Lima: Pontificia Universidad Católica del Perú.

Reimer, Joseph (1989). From Moral Discussion to Democratic Governance. En: Power, F. Clark; Higgins, Ann and Kohlberg, Lawrence. Lawrence Koblberg's Approach to Moral Education. Nueva York: Columbia University Press.

Tetlock, Philip E. (1983). Accountability and Complexity of Thought. Journal of Personality and Social Psychology, 45 (1), 74-85.

Tetlock, Philip E.; Bernzweig, J. \& Gallant, J.L. (1985). Supreme Court Decision Making: Cognitive Style as a Predictor of Ideological Consistency of Voting. Journal of Personality and Social Psychology, 48 (5), 1227-1239. 